\title{
Effects of Chemical Drive on the Inhibition of Respiration Produced by Laryngeal Stimulation in Cats
}

\author{
Takashi Nishino \\ Department of Physiology, School of Medicine Chiba Univ., \\ 1-8-1 Inohanacho, Chiba, 280 Japan
}

\begin{abstract}
In 8 anesthetized cats the acute respiratory responses to laryngeal stimulation were studied under various levels of chemical drive. Under air breathing condition with strong stimulation the animals showed either cough or apnea, whereas with relatively weak stimulation the animals showed smooth reduction in respiration due to a decrease in tidal volume and prolongation of both inspiratory and expiratory time. Although the inhibitory effect of laryngeal stimulation on tidal volume was independent of the level of chemical drive, the inhibition of respiration, when analyzed in terms of decrease in the minute ventilation, was greater the higher the chemical drive regardless of the type of chemical stimulus.

Although bilateral vagotomy decreased the threshold of stimulus intensity for changes in respiratory pattern, especially under hypoxia, the effects of laryngeal stimulation on respiratory pattern in vagotomized animals were qualitatively similar to those obtained in the intact animals. We concluded that laryngeal stimulation has a direct action on the central respiratory mechanism regulating respiratory duration and that the interaction between laryngeal stimulation and chemical drive causes multiplicative inhibitory effects on ventilation.
\end{abstract}

It is well known that the larynx and its associated structures are extremely sensitive sources of defense reflexes (WidDicombe, 1977). Chemical and mechanical stimulation of the laryngeal mucosa produces various changes in respiratory pattern (BousheY et al., 1972). Among these respiratory responses, inhibition of respiration is considered as one of the protective mechanisms with which the body can prevent further entry and penetration of an invading agent. Although it is well documented that laryngeal stimulation, including electrical stimulation of the superior laryngeal nerves, frequently results in a depression of respiration (Beyer, 1901; Gesell and Hamilton, 1941; Hammouda and Wilson, 1938), influences of the background chemical drive which determines the baseline respiratory activity on the inhibition of respiration during laryngeal stimulation

Received for publication May 14, 1980

西野 卓 
have seldom been evaluated. BousHeY et al. (1972) studied the reflex effects of mechanical and chemical stimulation on the pattern of breathing in air breathing cats (thus probably under normocapnic and normoxic conditions) and reported that inhibition of respiration was due to a decrease in tidal volume and prolongation of both inspiratory and expiratory time. However, they did not consider the influence of the background chemical drive on these changes in respiratory pattern. The purpose of this study is to examine the interaction of chemical drive with the inhibitory effects on respiration of laryngeal stimulation.

\section{METHODS}

Animal preparation. Eight adult cats (weighing 1.9-3.8 kg) were anesthetized with $\alpha$-chloralose (50 mg/kg given intraperitoneally). Tracheostomy was performed at the lower cervical trachea and the trachea was cannulated. Both vagosympathetic trunks were isolated and surrounded by loose ties for later sectioning. The recurrent laryngeal nerves were cut at the lower cervical level. Both femoral arteries were cannulated for the measurement of arterial blood pressure and for the withdrawal of arterial blood samples. A femoral vein was also cannulated. The rectal temperature of the animals was maintained at $37-$ $38^{\circ} \mathrm{C}$ with a heating lamp. A median cervical incision, directed rostrally until just below the cricoid cartilage, was made on the upper trachea and the larynx was exposed. Care was taken not to damage the superior laryngeal nerve during operation. An insulated monopolar electrode (TOP: specific resistance; 9.6 $\mu \Omega / \mathrm{cm}$ ) was placed on the mucosa of the posterior wall of the infraglottic cavity. The stimulation site was not changed throughout the experiment. An indifferent electrode was also placed on the posterior wall of the infraglottic cavity $4-5 \mathrm{~mm}$ apart from the stimulating electrode. Stimulation was always by square waves of $0.5 \mathrm{msec}$ duration and frequency of $25 \mathrm{~Hz}$, but the intensity was varied from 0 to $6.0 \mathrm{~V}$. Respiration was monitored with a pneumotachograph (Fleish $\# 00$ ) and the tidal volume was obtained by integration of inspired air flow signal. End-tidal $\mathrm{CO}_{2}\left(P_{\mathrm{ETCO}_{2}}\right)$ was monitored by a Beckman $\mathrm{CO}_{2}$ analyzer (LB-1). Esophagus pressure was also monitored. All the respiratory variables were continuously recorded on a visicorder (San-ei). Arterial blood samples were analyzed for $\mathrm{pH}, P_{\mathrm{CO}_{2}}$ and $P_{\mathrm{O}_{2}}$ immediately after sampling with a Radiometer blood gas analyzer (BM2 MK2).

Experimental procedures. All studies were performed with spontaneously breathing animals in the supine position. In order to obtain the threshold for changes in respiratory pattern, the intensity of electrical stimulation of the laryngeal mucosa was increased from 0 to $6.0 \mathrm{~V}$ with step increments of $0.2 \mathrm{~V}$ while the animal was quietly breathing room air. After obtaining the threshold value of stimulus intensity, the intensity was fixed at a certain level so that stimulation of the laryngeal mucosa caused smooth reduction in ventilation without causing cough or expiratory apnea. Then, the animal was subjected to steady- 
state inhalation of various concentrations of $\mathrm{CO}_{2}$ in oxygen or various concentrations of $\mathrm{O}_{2}$ in nitrogen. During inhalation of the hypoxic gas mixture, $\mathrm{CO}_{2}$ was added to the inspired line in order to maintain an isocapnic level of $P_{\mathrm{aCO}}$.

Following arterial blood sampling at each steady-state level, electrical stimulation of the laryngeal mucosa was induced during an expiratory period and maintained for the succesive two respiratory cycles. The baseline inspired minute ventilation $\left(\dot{V}_{\mathrm{I}}\right)$ was obtained from a 1-min recording of ventilation before laryngeal stimulation, and the inspired minute ventilation during laryngeal stimulation was calculated from the values of tidal volume and the corresponding respiratory duration of the first breath (instantaneous minute ventilation).

Immediately following the above experimental procedures, the animal was subjected to bilateral vagotomy, and the experiment of ventilatory responses to laryngeal stimulation under various conditions of chemical drive was repeated.

Following the above experiments, sectioning of the superior laryngeal nerves was performed in the region of the nodosa ganglion and respiratory response to laryngeal stimulation was tested.

\section{RESULTS}

Thresholds of stimulus intensity for inhibition of respiration and for cough or apnea reflex

No cats showed any change in on-going respiratory pattern with an electrical stimulation of less than $0.6 \mathrm{~V}$. With the increment of stimulus intensity to a certain level, sudden changes in respiratory pattern were observed in all the animals. Characteristic changes in respiratory pattern were a decrease in tidal volume and prolongation of both inspiratory and expiratory duration, as shown in Fig. 1 (a and c). With further increment of stimulus intensity, all the animals showed either coughing or apnea at some level of stimulus intensity, as seen in Fig.

Table 1. Thresholds of stimulus intensity for inhibition of respiration and for cough or expiratory apnea.

\begin{tabular}{cccccc}
\hline \multirow{2}{*}{ Cat No. } & \multicolumn{2}{c}{ Intact } & & \multicolumn{2}{c}{ Vagotomized } \\
\cline { 2 - 3 } \cline { 5 - 6 } & $T_{\text {in hib. }}(\mathrm{V})$ & $T_{\text {cough/apnea }}(\mathrm{V})$ & $T_{\text {in hib. }}(\mathrm{V})$ & $T_{\text {cough/apnea }}(\mathrm{V})$ \\
\hline 1 & 1.6 & 3.4 & & 1.0 & 2.0 \\
2 & 1.4 & 2.4 & & 1.0 & 0.8 \\
3 & 0.8 & 2.6 & & 0.6 & 1.6 \\
4 & 0.8 & 2.6 & & 0.6 & 1.8 \\
5 & 3.4 & 5.6 & & 1.2 & 3.0 \\
6 & 2.4 & 4.0 & & 1.8 & 3.0 \\
7 & 2.6 & 3.0 & & 0.6 & 2.2 \\
8 & 1.6 & $4.0 \pm 1.3$ & $1.0 \pm 04^{*}$ & $2.1 \pm 0.8^{*}$ \\
\hline Mean \pm SD & $1.8 \pm 0.9$ & &
\end{tabular}

Vol. 30, No. 6, 1980 


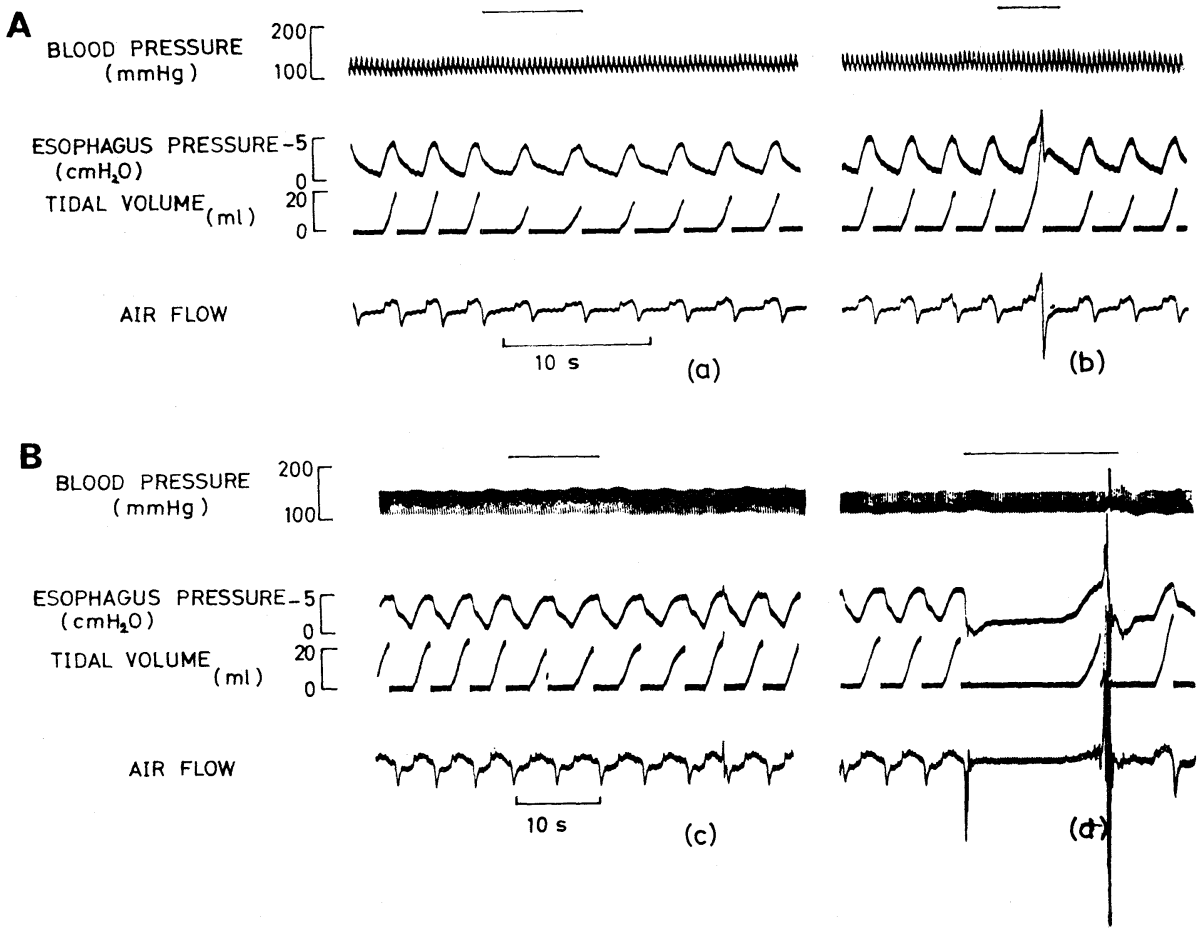

Fig. 1. Acute responses of respiration to electrical stimulation in two different cats (A and B). Weak stimulation $(\mathrm{a}, 0.8 \mathrm{~V} ; \mathrm{c}, 0.9 \mathrm{~V})$ caused smooth reduction in ventilation whereas strong stimulation (b, $2.6 \mathrm{~V} ; \mathrm{d}, 2.8 \mathrm{~V}$ ) caused coughing in A and apnea in B. Horizontal bars indicate the duration of electrical stimulation of the larynx.

1 ( $b$ and $d)$. These different responses to increasing levels of stimulus intensity were observed both before and after bilateral vagotomy. However, threshold values for changes in respiratory pattern decreased significantly after bilateral vagotomy (Table 1).

Interaction of laryngeal inhibitory reflex and chemical drive in the intact animals

In Fig. 2 the effects of laryngeal stimulation on respiration during hypercapnia and hypoxia are demonstrated. The changes in respiratory pattern characterized by a decrease in tidal volume and prolongation of both inspiratory and expiratory duration were observed even under hypercapnia and hypoxia. Figure 3 describes the $\mathrm{CO}_{2}$ response and the hypoxic response curves obtained in a cat before and during laryngeal stimulation. At all levels of $P_{\mathrm{a} \mathrm{O}_{2}}$ and $P_{\mathrm{aCO}_{2}}$ an inhibitory action of laryngeal stimulation on respiration was present. The $\mathrm{CO}_{2}$ response curve during laryngeal stimulation shifted downward with a reduction in slope. The same effect was observed in the hypoxic response curve during laryngeal stimulation. Figure 4 describes the differences in ventilation $\left(\Delta \dot{V}_{\mathrm{I}}\right)$ before and during 


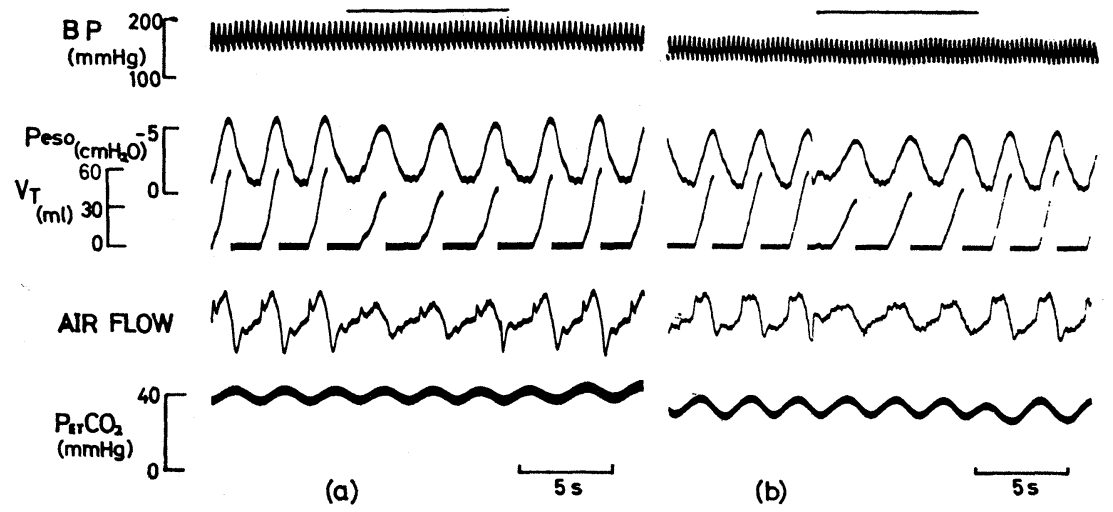

Fig. 2. Effects of laryngeal stimulation on respiration during hyperoxic hypercapnia (a) and normocapnic hypoxia (b). Horizontal bars indicate the duration of laryngeal stimulation.

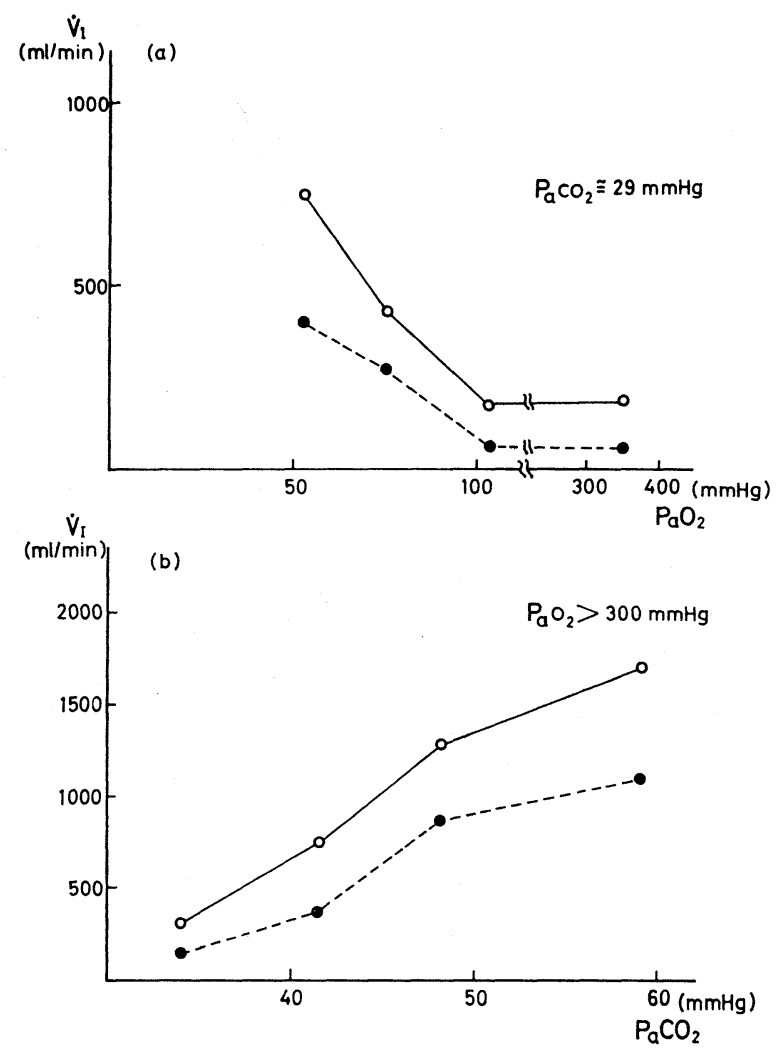

Fig. 3. The hypoxic response (a) and the $\mathrm{CO}_{2}$ response (b) curves before (solid line) and during (broken line) laryngeal stimulation obtained from an intact cat. 

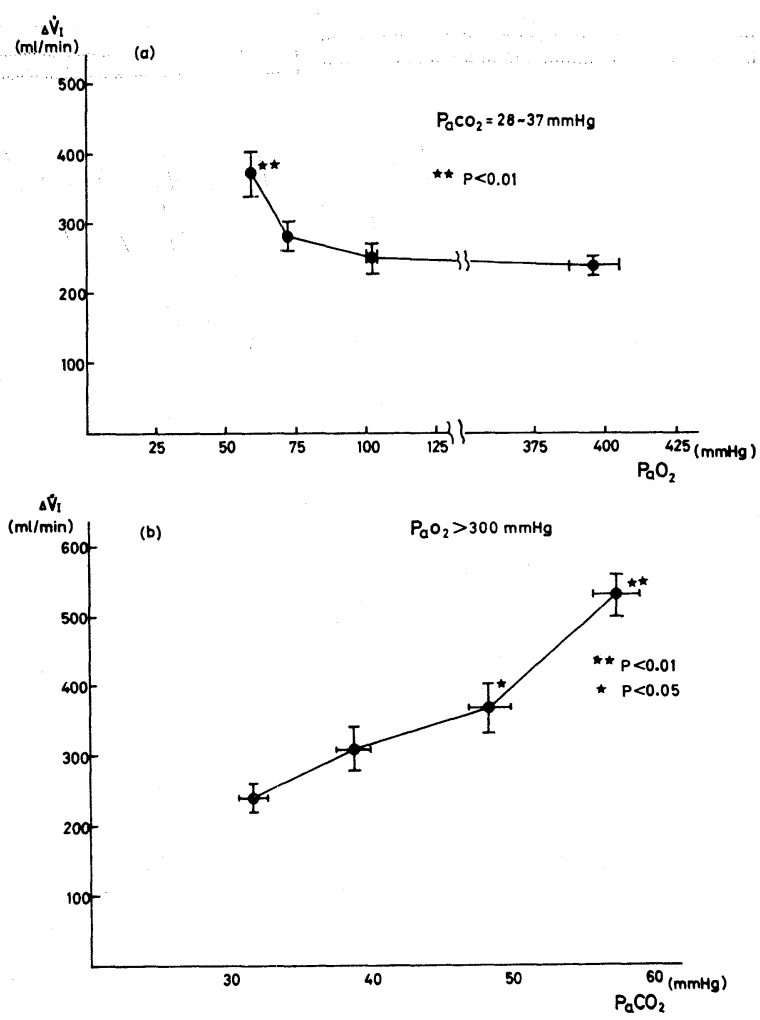

Fig. 4. Differences of ventilation between before and during laryngeal stimulation at various levels of chemical drive ( $n=8$, values represent mean $\pm \mathrm{SE})$.

laryngeal stimulation at various levels of $P_{\mathrm{aO}_{2}}$ and $P_{\mathrm{aCO}}$. There was a significant increase in $\Delta \dot{V}_{\mathrm{I}}$ at higher levels of chemical drive, regardless of whether the chemical drive was hypoxia or hypercapnia. Thus, inhibition of respiration during laryngeal stimulation appears to be dependent on the background chemical drive. Figure 5 shows the relationship between tidal volume and chemical drive, and the relationship between respiratory frequency and chemical drive in the same cat as in Fig. 3. It can be seen that laryngeal stimulation shifted the curves for these relationships downward in a parallel fashion. The decreases in tidal volume and respiratory frequency caused by laryngeal stimulation at various levels of chemical drive for 8 different cats are shown in Fig. 6. These results indicate that decreases in tidal volume and respiratory frequency are independent of chemical drive levels and that laryngeal stimulation and chemical drive cause simply additive inhibitory effects on tidal volume and respiratory frequency. These findings also suggest that the inhibition of respiration during laryngeal stimulation is independent of the type of background chemical stimulus. 

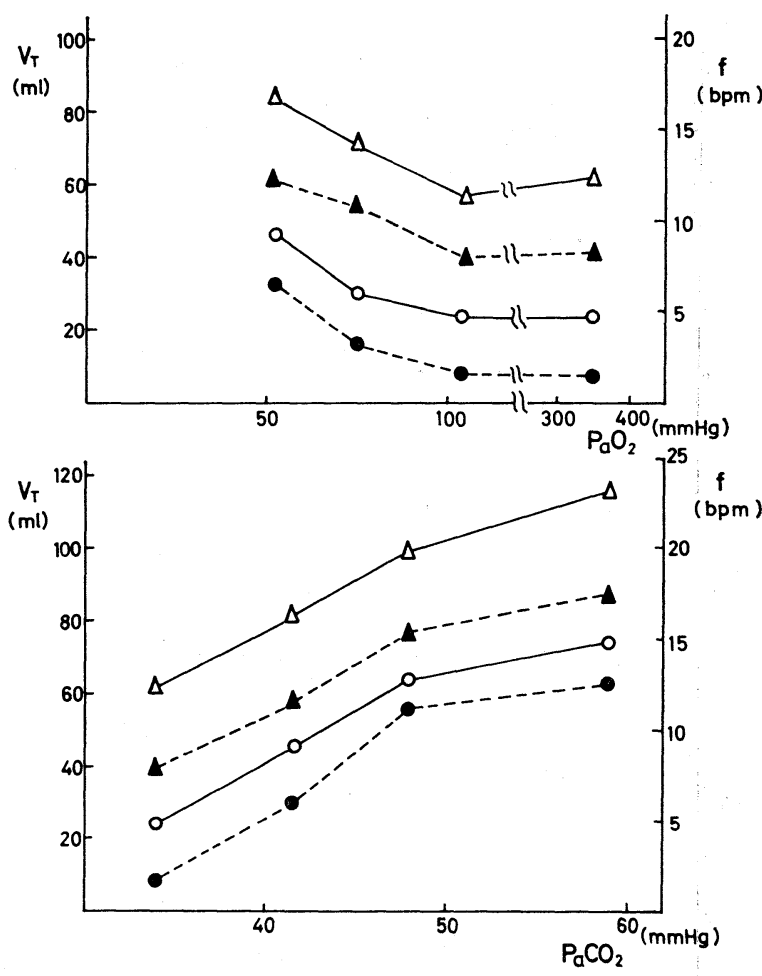

Fig. 5. Effects of increasing levels of chemical drive on tidal volume and respiratory frequency before and during laryngeal stimulation obtained in the same cat as in Fig. 3 . Tidal volume before (open circles) and during (closed circles) laryngeal stimulation, and respiratory frequency before (open triangles) and during (closed triangles) laryngeal stimulation are plotted against increasing levels of chemical drive.

$V_{\mathrm{T}}$ vs. $T_{\mathrm{I}}$ relationship in the intact animals

In order to evaluate the effects of inhibito-inspiratory Hering-Breuer reflex before and during laryngeal stimulation the relationships between tidal volume $\left(V_{\mathrm{T}}\right)$ and inspiratory time $\left(T_{\mathrm{I}}\right)$ in response to an increase in chemical drive were plotted. As shown in Fig. 7 the $V_{\mathrm{T}}$ vs. $T_{\mathrm{I}}$ relationship during laryngeal stimulation shifted to the right without changing its hyperbolic shape. Similar changes in $V_{\mathrm{T}}$ vs. $T_{\mathrm{I}}$ relationships in response to changes in $P_{\mathrm{a}_{2}}$ and $P_{\mathrm{aCO}_{2}}$ were observed in all animals. These results suggest that the reduction in respiratory frequency during laryngeal stimulation results not only from the decreased sensory vagal input caused by the decrease in tidal volume, but also from the direct action of laryngeal stimulation on the central respiratory mechanism.

$T_{\mathrm{I}}$ vs. $T_{\mathrm{E}}$ relationship in the intact animals

Increased chemical drive, whether hypercapnia or hypoxia, decreased both 

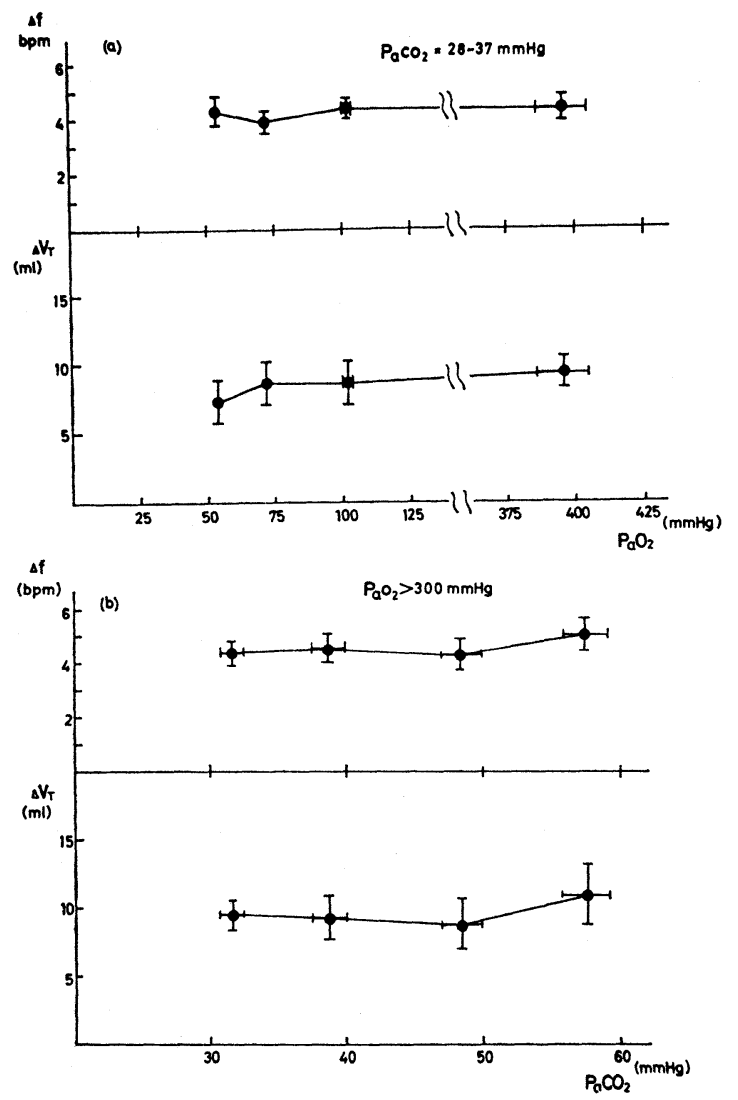

Fig. 6. Differences of tidal volume and respiratory frequency at various levels of chemical drive $(n=8$, values represent mean $\pm \mathrm{SE})$.

inspiratory $\left(T_{\mathrm{I}}\right)$ and expiratory time $\left(T_{\mathrm{E}}\right)$. Figure $8(\mathrm{a}$ and $\mathrm{b})$ shows the typical $T_{\mathrm{I}}$ vs. $T_{\mathrm{E}}$ relationships in response to an increase in $P_{\mathrm{acO}_{2}}$ and a decrease in $P_{\mathrm{aO}_{2}}$. Before laryngeal stimulation the $T_{\mathrm{I}}$ vs. $T_{\mathrm{E}}$ relationships were linear. On the other hand, the $T_{\mathrm{I}}$ vs. $T_{\mathrm{E}}$ relationships during laryngeal stimulation were curvilinear.

\section{Effects of bilateral vagotomy}

After vagotomy an increased sensitivity to electrical stimulation of the laryngeal mucosa was observed (Table 1). This effect was most prominent under the hypoxic condition. The same stimulus intensity as given to the intact animal caused apnea in 5 of 8 cats under hypoxia, whereas under hyperoxia, 2 of 8 cats showed apnea. An example of long-lasting apnea observed under hypoxia is shown in Fig. 9. For this reason we could not obtain the full range of hypoxic response and $\mathrm{CO}_{2}$ response curves from all animals. However, the obtained hy- 


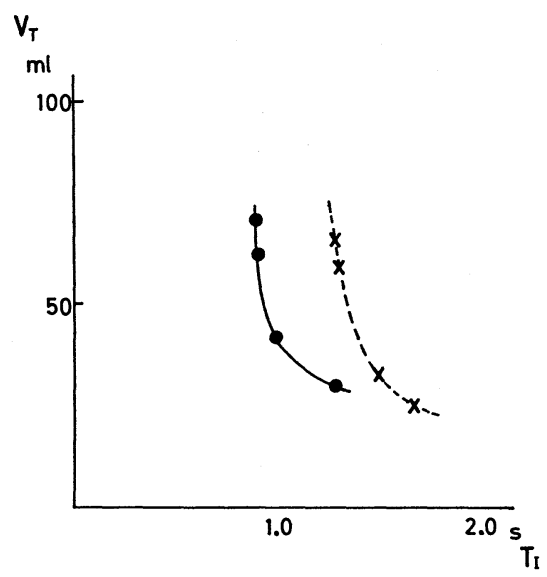

Fig. 7. The $V_{\mathrm{T}}$ vs. $T_{\mathrm{I}}$ relationship before (closed circles) and during (crosses) laryngeal stimulation in the intact cat.
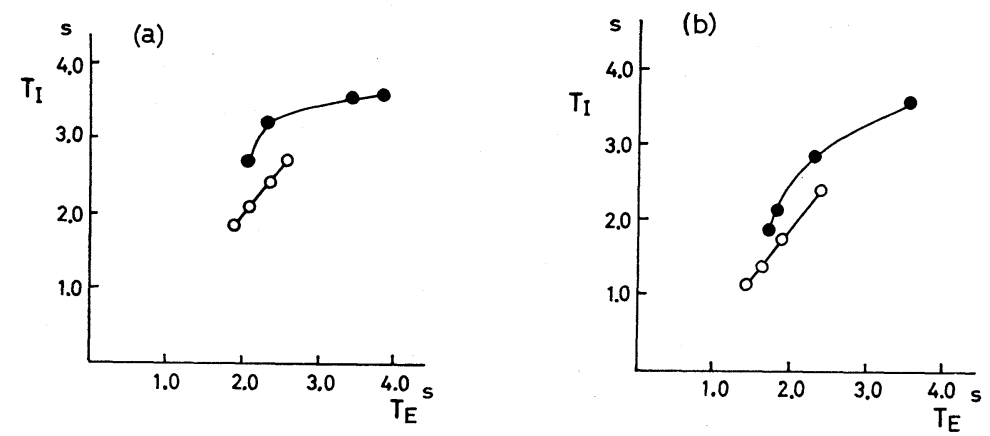

Fig. 8. The $T_{\mathrm{I}}-T_{\mathrm{E}}$ relationship in response to an increase in $P_{\mathrm{acO}_{2}}$ (a) and a decrease in $P_{\mathrm{aO} 2}(\mathrm{~b})$ before (open circles) and during (closed circles) laryngeal stimulation.

poxic response and $\mathrm{CO}_{2}$ response curves showed that the effects of laryngeal stimulation were qualitatively similar to those in the intact animals (Fig. 10). Thus, the $\mathrm{CO}_{2}$ response and the hypoxic response curves shifted downward with a growing tendency towards a decrease in slope at a higher chemical drive during laryngeal stimulation. The reduction in respiration was due to a decrease in tidal volume and prolongation of both inspiratory and expiratory time. An example of the $V_{\mathrm{T}}$ vs. $T_{\mathrm{I}}$ relationship is shown in Fig. 11. Like the responses in the intact animals, the parallel shifts of the $V_{\mathrm{T}} v s . T_{\mathrm{I}}$ relationship were observed during laryngeal stimulation although the hyperbolic relationship observed before vagotomy was not observed after vagotomy. This confirms that the laryngeal stimulation has a direct action on the central respiratory mechanism which regulates the respiratory duration. 


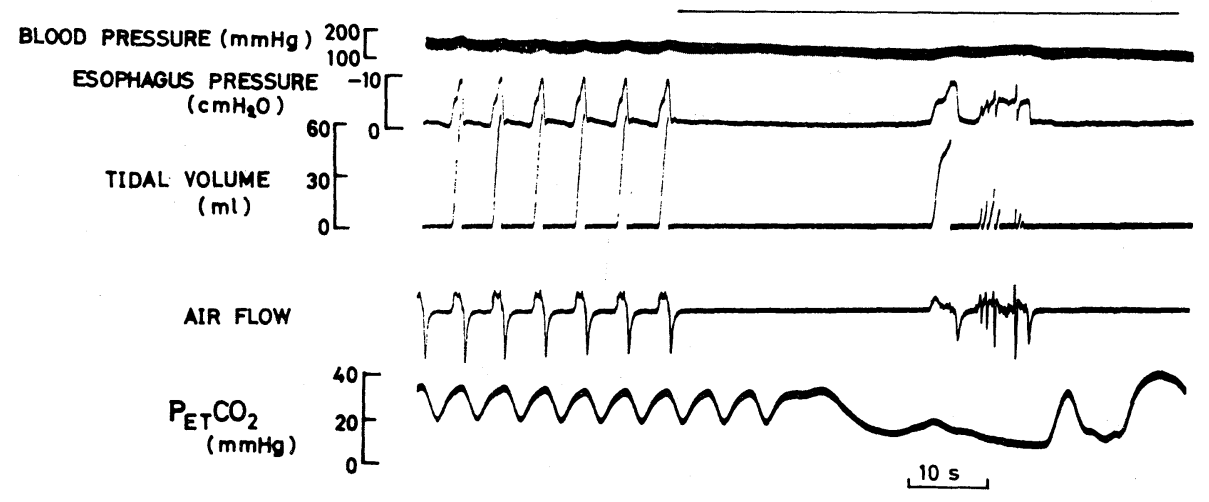

Fig. 9. Effects of laryngeal stimulation on ventilation under hypoxia in a spontaneously breathing, vagotomized cat. Horizontal bar represents the period of laryngeal stimulation. The values of $\mathrm{pH}, P_{\mathrm{acO}_{2}}$ and $P_{\mathrm{aO} 2}$ before laryngeal stimulation are $7.340,34$ $\mathrm{mmHg}$ and $50 \mathrm{mmHg}$, respectively.

\section{Effects of the superior laryngeal nerve resection}

Sectioning of the superior laryngeal nerves abolished the effects of laryngeal stimulation in all the animals except one in which a slight reduction in respiration was still observed with a strong stimulation of the laryngeal mucosa.

\section{DISCUSSION}

We demonstrated in this study that a relatively weak electrical stimulation of the laryngeal mucosa can inhibit respiration without causing cough or spasmodic respiratory movement, whereas a stronger stimulation frequently caused coughing or gasp-like breathing or sudden apnea. Facilitation of respiration during laryngeal stimulation, described by other investigators (HILLENBRAND and BOYD, 1936; Hukuhara, personal communication), was not observed in this study, possibly due to the full level of anesthesia employed in this study (HAMmouda and WiLson, 1938). Our observations are in agreement with the report of BousHEY and others (1972), who stimulated the laryngeal mucosa mechanically or chemically and showed that strong stimulation commonly produced coughing or gasping, whereas weak stimulation resulted in only a slight inhibition of respiration. Although we did not determine the nature of receptors responsible for these actions, the difference between strong and weak stimulation may be the effect of central response to different intensities of stimulation from the same population of receptors or it may be the effect of central response to stimulation of different sets of receptors.

The superior laryngeal nerve has been accepted as the principal ner ve supply to the larynx (Pressman and Kelemen, 1955). Since both sides of the recurrent laryngeal nerves were cut in this study and, moreover, resection of both sides of the superior laryngeal nerves eliminated all the reflex actions in most of the animals, the superior laryngeal nerve was considered principally to be responsible 


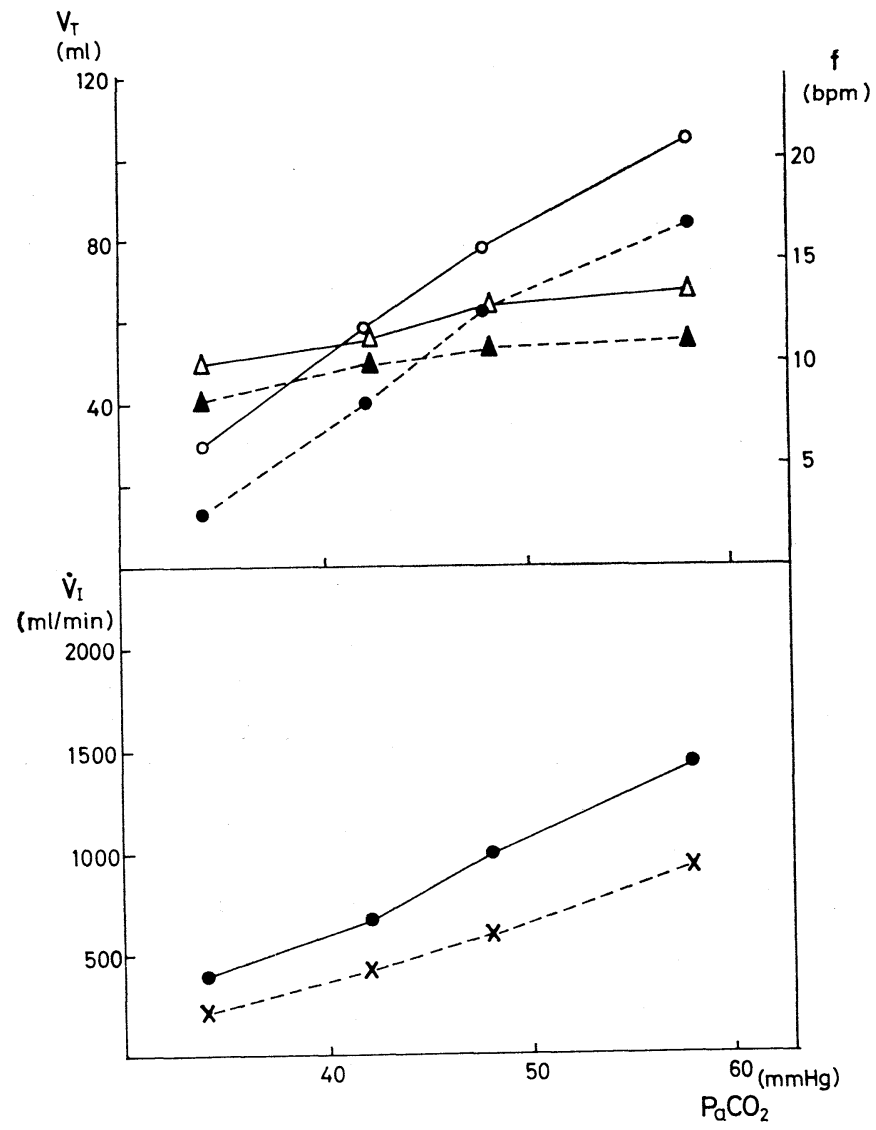

Fig. 10. Effects of increasing $P_{\mathrm{aco} 2}$ on tidal volume, respiratory frequency and ventilation before (solid lines) and during (broken lines) laryngeal stimulation. In the upper panel circle symbols and triangle symbols represent tidal volume and respiratory frequency, respectively. In the lower panel closed circles and crosses represent ventilation before and during laryngeal stimulation, respectively.

for the reflex actions induced by electrical stimulation of the laryngeal mucosa in this study.

Changes in respiratory pattern under various levels of chemical drive. Reduced ventilation during laryngeal stimulation was due to a decrease in tidal volume and prolongation of both inspiratory and expiratory time. These changes in respiratory pattern occurred under both hypoxic and hypercapnic conditions and the type of chemical stimulus made no difference. Reductions in tidal volume and respiratory frequency during laryngeal stimulation were independent of chemical drive, and therefore, laryngeal stimulation and chemical drive were considered to be additive stimuli with respect to tidal volume and respiratory frequency. However, when respiratory activity was analyzed in terms of the minute ventila- 


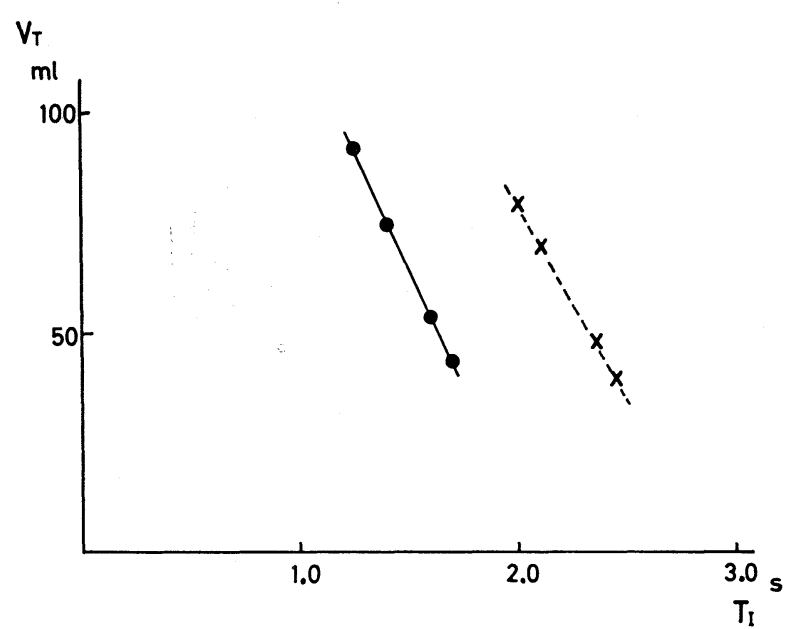

Fig. 11. The $V_{\mathrm{T}}$ vs. $T_{\mathrm{I}}$ relationship before (closed circles) and during (crosses) laryngeal stimulation in the vagotomized cat.

tion which represents the product of tidal volume and respiratory frequency, both $\mathrm{CO}_{2}$ response and hypoxic response were decreased during laryngeal stimulation. This indicates that laryngeal stimulation and chemical drive act as multiplicative stimuli on ventilation. It may be misleading, however, to consider this effect as multiplicative ventilatory interaction if the reduction in respiratory frequency is dependent solely on the concomitant changes in tidal volume (inhibito-inspiratory Hering-Breuer reflex effect represented by the hyperbolic $V_{\mathrm{T}} v s . T_{\mathrm{I}}$ relationship: Clark and Euler, 1972; Grunstein et al., 1973). By plotting the relationship between $V_{\mathrm{T}}$ and $T_{\mathrm{I}}$, we found that in the intact animals the laryngeal stimulation shifted the $V_{\mathrm{T}} v s$. $T_{\mathrm{I}}$ relationship to the right without changing the characteristic hyperbolic shape. The parallel shift of the $V_{\mathrm{T}} v s . T_{\mathrm{I}}$ relationship was observed after vagotomy. These results indicate that the threshold for inspiratory off-switch is elevated by a direct action of laryngeal stimulation and that the reduction in respiratory frequency is not only due to a secondary effect of decreased tidal volume but also due to direct action of laryngeal stimulation on the central mechanism regulating respiratory duration. Thus, it may be concluded that as far as the ventilation is concerned, there is a multiplicative interaction between laryngeal stimulation and chemical drive.

Our results for the $T_{\mathrm{I}}-T_{\mathrm{E}}$ relationship in the intact animals confirmed the finding of CLARK and EULER (1972) that $T_{\mathrm{I}}$ and $T_{\mathrm{E}}$ are linearly related when ventilation is increased by elevated chemical drive. During laryngeal stimulation, however, $T_{\mathrm{I}}$ tended to be disproportionately large at higher levels of chemical drive and the $T_{\mathrm{I}}-T_{\mathrm{E}}$ relationship was curvilinear. This effect may be a common feature of response to sudden change in stimulus since a similar non-linear $T_{\mathrm{I}}-T_{\mathrm{E}}$ relationship was reported by WIDDICOMBE and WINNING (1974), who tested the 
effect of sudden changes in ventilation on the $T_{\mathrm{I}}-T_{\mathrm{E}}$ relationship. Although both $T_{\mathrm{I}}$ and $T_{\mathrm{E}}$ are prolonged by laryngeal stimulation after vagotomy, the $T_{\mathrm{I}}-T_{\mathrm{E}}$ relationship was variable and no consistent relationship was observed. Thus, the characteristic curvilinear $T_{\mathrm{I}}-T_{\mathrm{E}}$ relationship during laryngeal stimulation appeared to be dependent on integrity of the vagus nerves.

Changes in respiratory pattern in the vagotomized animals. Perhaps the most surprising result was that the inhibitory action of laryngeal stimulation was augmented after vagotomy. All the animals clearly showed a decrease in threshold values for changes in respiratory pattern indicating that vagal afferents have some inhibitory influence on the laryngeal reflexes. It appeared that hypoxia further enhanced this augmented reflex effect due to vagotomy since more than half of the vagotomized animals showed apnea under hypoxia with the same stimulus intensity that caused slight inhibition of respiration in the intact animals. The mechanism of this action is not clear. This augmented laryngeal reflex effect under hypoxia after vagotomy may be analogous to the exaggerated hypoxic response in decorticate animals reported by TENNEY and Ou (1977). They emphasized the importance of the inhibitory descending influence from the cerebrum in the control of breathing. BouSHEY and RICHARDSON (1973) studied the reflex effects on the pattern of breathing by introducing high concentrations of $\mathrm{CO}_{2}$ into the larynx in anesthetized and decerebrated cats. They showed that the average strength of the reflex was greater in decerebrated than in anesthetized cats. Although they attributed their finding to the depressive effect of anesthetics on the reflex pathway or to the difference of mucous production activity between anesthetized and decerebrated cats, it may also be possible to consider the inhibitory descending influence from the higher central nervous system. Possibly, decerebration could eliminate the inhibitory descending influence and the augmented reflex resulted in. Whatever the mechanism may be, it is clear that absence of vagal afferents plays an important role in causing apnea under hypoxia. In this regard, it is interesting to note that primary afferent fibers in the superior laryngeal nerves and in the vagus synapse onto neurons of the nucleus tractus solitarius in which termination of carotid body chemoreceptor afferents are located (Cottle, 1964; Davis and Edwards, 1973). cussion.

I am grateful to Dr. Y. Honda, Dr. Y. Fukuda, and Dr. T. Yonezawa for advice and dis-

\section{REFERENCES}

Beyer, H. (1901) Athemreflexe auf olfactoriuseiz. Arch. Anat. Physiol. (Suppl.), 261-275.

Boushey, H. A., Richardson, P. S., and Widdicombe, J. G. (1972) Reflex effects of laryngeal irritation on the pattern of breathing and total lung resistance. J. Physiol. (Lond.), 224: 501-513.

Boushey, H. A. and Richardson, P.S. (1973) The reflex effects of intralaryngeal carbon dioxide on the pattern of breathing. J. Physiol. (Lond.), 228: 181-191.

Vol. 30, No. 6, 1980 
Clark, F. J. and Von Euler, C. (1972) On the regulation of depth and rate of breathing. $J$. Physiol. (Lond.), 222: 267-295.

Cottle, M. K. (1964) Degeneration studies in primary afferents of IXth and Xth cranial nerves in the cat. J. Comp. Neurol., 122: 329-343.

Davis, R. O. and EDwards, M. W. (1973) Distribution of carotid body chemoreceptor afferents in the medulla of the cat. Brain Res., 64: 451-454.

Gesell, R. and Hamilton, M. A. (1941) Reflexogenic components of breathing. Am. J. Physiol., 133: 694-719.

Grunstein, M. M., Younes, M., and Millic-Emilli (1973) Control of tidal volume and respiratory frequency in anesthetized cats. J. Appl. Physiol., 35: 463-476.

Hammouda, M. and Wilson, W. H. (1938) Reflex acceleration of respiration arising from excitation of the vagus or its terminations in the lungs. J. Physiol. (Lond.), 94: 497-524.

Hillenbrand, G. J. and Boyd, T. E. (1936) Reflex respiratory effects from intermittent stimulation of the vagus and superior laryngeal nerves. Am. J. Physiol., 116: 380-386.

Pressman, J. J. and Kelemen, G. (1955) Physiology of the larynx. Physiol. Rev., 35: 506-554.

TenneY, S. M. and Ou, L. L. (1977) Ventilatory response of decorticate and decerebrate cats to hypoxia and $\mathrm{CO}_{2}$. Respir. Physiol., 29: 81-92.

Widdicombe, J. G. and Winning, A. (1974) Effects of hypoxia, hypercapnia and changes in body temperature on the pattern of breathing in cats. Respir. Physiol., 21: 203-221.

Widdicombe, J. G. (1977) Respiratory reflexes and defense. In: Respiratory Defense Mechanism, Part II, ed. by Brain, J. D., Proctor, D. F., and ReID, L. Mercel Dekkar, New York, pp. 593-630. 